\title{
Effects of Different Inoculated Pathogens on Microbial Diversity of Rhizosphere Soil of Tobacco in Marigold-Tobacco Intercropping System
}

\author{
Li Lin ${ }^{1, ~ a, ~ B i n g ~} \mathrm{Li}^{2}$, Zebin Chen ${ }^{1}$, Yujiao Zhu' ${ }^{3}$, Yuan Su${ }^{4}$, Lei Yu ${ }^{5}$, Shengguang $\mathrm{Xu}^{6,}$, , \\ ${ }^{1}$ College of Agronomy, Kunming University, Kunming, Yunnan Province, China. \\ ${ }^{2}$ Kunming Institute of Animal Research, Chinese Academy of Sciences, Kunming, Yunnan Province, China. \\ ${ }^{3}$ Affiliated Middle School of Yunnan Agricultural University, Kunming, Yunnan Province, China. \\ ${ }^{4}$ Key Laboratory of Characteristic Biological Resource Development and Utilization of Colleges and \\ Universities in Yunnan Province, Kunming, Yunnan Province, China. \\ ${ }^{5}$ Yunnan Urban Characteristic Agriculture Engineering Technology Research Center, Kunming, Yunnan \\ Province, China. \\ ${ }^{6}$ Yunnan Institute of Biological Carbon Engineering Research Center, Kunming, Yunnan Province, China. \\ allysj4809@163.com; bsgxu2011@126.com \\ *Corresponding author: Shengguang Xu
}

Keywords: tobacco; Marigold; rhizosphere soil; high throughput sequencing; microbial diversity

\begin{abstract}
The aim of this research is to clarify the effects of different inoculated pathogens on the microbial diversity of rhizosphere soil of tobacco in marigold-tobacco intercropping system. The DNA of 16S rDNA-V4 region and ITS1 region in the rhizosphere soil of tobacco are sequenced with high throughput sequencer. The results show that after inoculating different pathogens, the abundances of Actinobacteria and Gemmatimonadetes increase; the abundance of Ascomycota decrease. In the tobacco-marigold intercropping system, the abundances of Actinobacteria, Gemmatimonadetes and Ascomycota increase. By inoculating different pathogens in tobacco plants in the marigold-tobacco intercropping system, and analyzing changes in microbial flora of the rhizosphere soil, this paper could provide a basis for analyzing the nematode killing mechanism of marigold.
\end{abstract}

\section{Introduction}

Marigold (Tagetes Erecta) is the earliest known plant which can control the nematode in soil and has development or application value. Many researchers have analyzed the mechanism of marigold in killing nematode. It is generally believed that the root exudates of marigold can kill nematodes or create soil ecological environment which is conducive to control microorganisms, and then control parasitic nematodes. For example, Huan-yu Wei and others found that the intercropping of marigold and Angelica could effectively control pathogenic fungi in the soil. [9] Sheng-jun Li found that the marigold-onion intercropping could improve the microclimate of fields, enhance the permeability and play ecological effects. [1] Lan-hong Jia and others found that the intercropping of marigold and dwarf, time difference economic crops can effectively control the disease epidemic. [2,3] Qian-jing Huang found the intercropping of marigold, wheat and cantaloupe could reduce the harm of diseases and insects, improve land use rate and increase farmers' income. [4,5] In this study, different pathogens were inoculated in the marigold-tobacco intercropping system. The DNA of 16S rDNA-V4 region and ITS1 region in tobacco rhizosphere soil after inoculation were sequenced by high throughput sequencing technology. Microorganisms in the rhizosphere soil of tobacco were studied by comparing soil samples from single tobacco planting groups and non-inoculation groups. The changes of floristic elements were preliminarily clarified to analyze the disease prevention mechanism of marigold-tobacco intercropping system, and provided a theoretical basis for the future 
intercropping of marigold with other crops.

\section{Research Materials and Methods}

\subsection{Test plants}

The tested tobacco was Yunyan 87. Plants were transplanted in the field of Nanyuan, Kunming Institute from 22 to 24 April, 2016. Three marigold seeds were sown near the root of tobacco plants. The pathogens of black shank and root knot nematode were inoculated at the seedling stage. The single tobacco planting and clean water procedure were taken as the control group.

\subsection{Pathogen inoculation}

Pathogens were provided by the plant protection research group of the College of Agronomy. The root damage perfusion method was used to inoculate the flue-cured tobacco. First, a small amount of sterile water was used to moisten the culture soil; then sterilized forceps were inserted deeply around the tobacco plant to achieve the effect of root injury. The $1 \times 104 \mathrm{cfu} / \mathrm{mL}$ zoospore suspension liquid was used to inoculate tobacco plants at 6-8 stages; each plant was inoculated with $10 \mathrm{~mL}$ suspension liquid. Root knot nematode was obtained from the root knot nematode infected tobacco plants in the root knot nematode nursery of Yunnan Academy of Tobacco Agricultural Science and Experimental Base. The soil was washed away from the root of infected tobacco plants; the egg sacs were removed with tweezers, and then put into the $1 \%$ sodium hypochlorite for 3 minutes to sterilize. Then egg sacs were washed with sterile water for 3 times, and placed in petri dishes with a small amount of aseptic water at 25 degrees centigrade. Hatched root knot nematodes were collected every 24 hours, and the nematode suspension was put into the sterilized triangle bottles with aseptic water. The concentration was about 2000 nematode $\cdot \mathrm{mL}^{-1}$. After the seedlings were transplanted for 3 days, each plant was irrigated with $200 \mathrm{~mL}$ suspension.

\subsection{Soil sample collection}

In the squaring stage of tobacco, the "S" sampling method was used to collect soil samples from the rhizosphere soil of tobacco plants at the depth of $5-20 \mathrm{~cm}$. The mixed soil samples were packed in sterile plastic bags, sealed and brought back to the laboratory for refrigeration at $4^{\circ} \mathrm{C}$. Soil samples of single cropping tobacco plants were recorded as AB3.2; soil samples inoculated with root knot nematodes of single cropping tobacco plants were recorded as AG3.2; soil samples inoculated with black shank pathogens of single cropping tobacco plants were recorded as AH3.2. Intercropping tobacco soil samples were recorded as BB3.2; intercropping tobacco soil samples inoculated with root knot nematode were recorded as BG3.2; intercropping tobacco soil samples inoculated with black shank pathogenic bacteria were recorded as BH3.2.

\subsection{Extraction of total DNA}

The E.Z.N.A. Soil DNA Kit genomic DNA extraction kit was used to extract soil DNA according to the instruction manual.

\subsection{PCR amplification}

16S rDNA-V4 and ITS1 region specific primers with Barcode were used to carry out PCR with high efficiency and high fidelity enzyme. The amplified PCR products were detected by agarose gel electrophoresis and sent to Beijing Genome Sequencing Company for high throughput sequencing.

\subsection{Bioinformatics analysis}

First of all, the effective data (effective tags) was obtained after splicing original data with Flash software, filtering the data with Qiime software, and removing chimera with Uchime Algorithm software. Greengene database was used in plant species annotation to find the species composition of microbial community. 


\section{Research Results and Analysis}

\subsection{Analysis on the characteristics of bacterial community composition}

The proportions of dominant bacteria in different soil samples were significantly different (Figure. 1). The proportions of Alcanivorax in soil samples of AB3.2 and AH3.2 were $1.75 \%$ and $0.92 \%$ respectively; in the soil samples of AG3.2 and BG3.2 the proportions were almost zero; the highest proportion in marigold intercropping soil samples was $0.39 \%$. Marinobacter was not contained in AG3.2 and BG3.2, but in other soil samples, the highest proportion was $3.39 \%$ in AB3.2; the proportions of Pseudomonas in soil samples of AG3.2 and BG3.2 were lower than that of other soil samples. The bacteria with difference greater than $1 \%$ between AB3.2 and AG3.2 were Sphingomonas, Marinobacter and Alcanivorax. The bacterium with difference greater than $1 \%$ between AG3.2 and BG3.2 was Sphingomonas. The bacteria with difference greater than $1 \%$ between BG3.2 and BB3.2 were Marinobacter and Pseudomonas. The bacterium with difference greater than 1\% between AB3.2 and BB3.2 was Alcanivorax. The bacteria with difference greater than 1\% between AB3.2 and AH3.2 were Marinobacter and Alcanivorax. The differences between proportions of dominant bacteria in soil samples of AH3.2, BH3.2 and BB3.2 were not obvious. Compared the single cropping group infected with root knot nematode with the non-inoculation single cropping group, the proportions of Sphingomonas, Marinobacter and Alcanivorax decreased; the proportions of Gemmatimonas, Blastococcus, Streptomyces and Arthrobacter increased. Compared the intercropping group inoculated with root knot nematode with single cropping group inoculated with root knot nematode, the proportions of Marinobacter and Alcanivorax decreased, while the proportions of Sphingomonas, Gemmatimonas, Blastococcus, Streptomyces and Arthrobacter increased. Compared the single cropping group infected with black shank pathogen with the non-inoculation single cropping group, the proportions of Sphingomonas, Gemmatimonas and Pseudomonas increased. Compared the inter cropping group infected with black shank pathogen with the single cropping group infected with black shank pathogen, the proportions of Sphelomonas, Gemmatimonas and Pseudomonas increased, while the proportion of Alcanivorax decreased. In general, the inoculation of different pathogens and the intercropping with marigold increased the number and richness of bacteria species. The number and richness of bacteria species in the intercropping system inoculated with different pathogens were higher than those of the non-inoculation and single cropping groups.

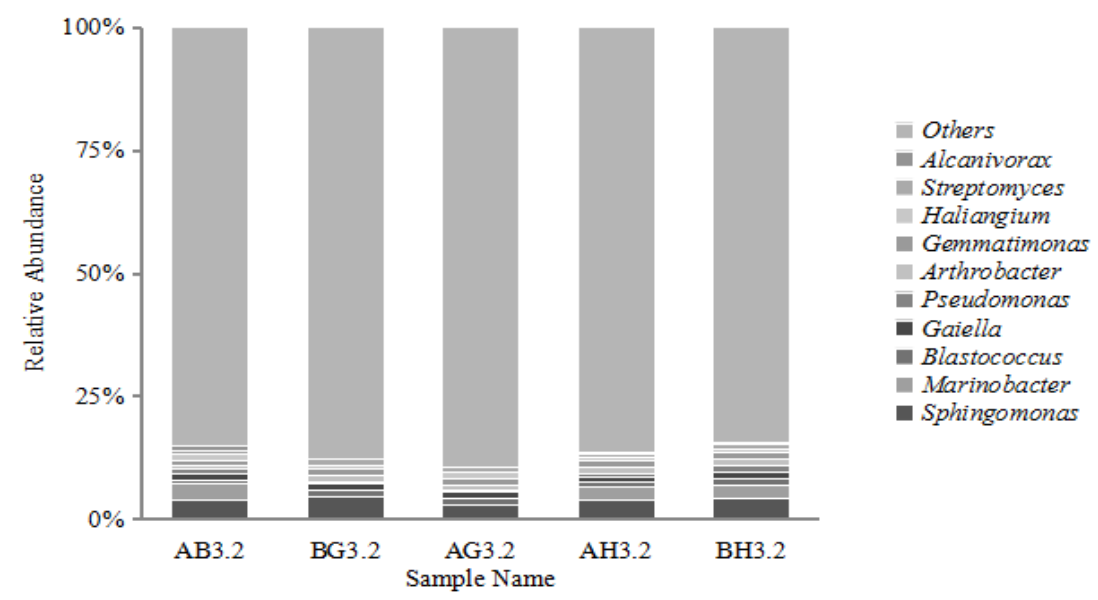

Figure. 1 Columnar diagram of relative abundance of bacteria species at horizontal level

\subsection{Analysis on the characteristics of fungi community composition}

The proportions of dominant fungi in different soil samples were significantly different. The proportion of Auxarthron in BG3.2 soil samples was 8.06\%; in other soil samples the proportions were almost zero. Aspergillus accounted for only 3.82\% of the AG3.2 soil sample, while in other soil samples, it accounted for more than 7\%. The proportion of Mortierella in AH3.2 soil sample was 
9.95\%, while the highest proportion in other soil samples was only $4.03 \%$. The fungi with difference greater than 1\% in AB3.2 and AG3.2 were Chaetomium, Aspergillus, Cryptococcus, Penicillium, Myrothecium and Humicola. The fungi with difference greater than 1\% in AG3.2 and BG3.2 were Chaetomium, Aspergillus, Auxarthron, Penicillium and Myrothecium. The fungi with difference greater than $1 \%$ in BG3.2 and BB3.2 were Chaetomium, Aspergillus, Mortierella, Auxarthro, Penicillium and Myrothecium. The fungi with difference greater than 1\% in AB3.2 and BB3.2 were Chaetomium, Aspergillus, Mortierella, Cryptococcus, Penicillium, Myrothecium and Humicola. The fungi with difference greater than $1 \%$ in AB3.2 and AH3.2 were Mortierella, Cryptococcus and Humicola. The fungi with difference greater than 1\% in AH3.2 and BH3.2 were Chaetomium, Aspergillus, Mortierella and Myrothecium. The fungi with difference greater than 1\% in BH3.2 and BB3.were Mortierella and Myrothecium. In the experiments of inoculating root knot nematodes, the proportions of Colletotrichum, Myrothecium, Humicola and Staphylotrichum increased; the proportion of Aspergillus decreased. In experiments of inter cropping marigold, the results were the opposite. Auxarthron was discovered for the first time and might become a new biocontrol bacterium. In the tests of inoculating black shank pathogen, the proportions of Aspergillus from high to low were AB3.2 > AH3.2 > BH3.2. The proportion of Mortierella in the soil sample of AH3.2 was up to 9.95\%, far greater than the proportions of other soil samples. The differences in the proportions of other dominant fungi were not significant.

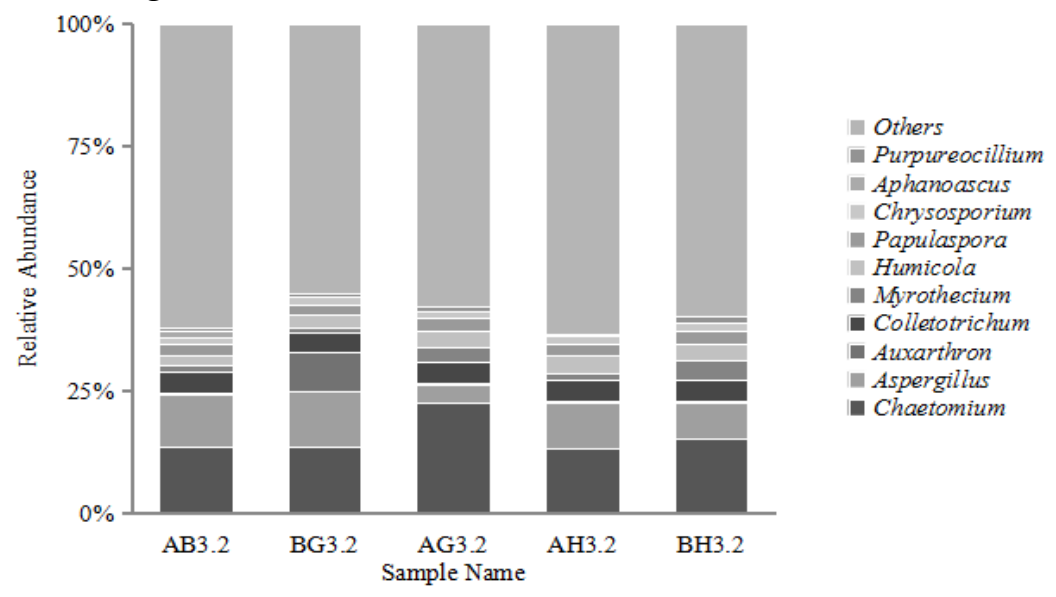

Fig. 2 Columnar diagram of relative abundance of fungi species at horizontal level

\section{Conclusion and Discussion}

In this study, the DNA of 16S rDNA-V4 region and ITS1 region in rhizosphere soil of tobacco were sequenced through high throughput sequencing technology. Results showed that microbial flora in the rhizosphere soil of tobacco was extremely rich. Sequence comparison revealed that the dominant bacteria found in the six soil samples were Proteobacteria, Actinobacteria and Gemmatimonadetes. In the dominant bacteria, compared the single cropping group infected with root knot nematode with the non-inoculation single cropping group, the proportions of Sphingomonas, Marinobacter and Alcanivorax decreased; the proportions of Gemmatimonas, Blastococcus, Streptomyces and Arthrobacter increased. Compared the intercropping group inoculated with root knot nematode with single cropping group inoculated with root knot nematode, the proportions of Marinobacter and Alcanivorax decreased, while the proportions of Sphingomonas, Gemmatimonas, Blastococcus, Streptomyces and Arthrobacter increased. Compared the single cropping group infected with black shank pathogen with the non-inoculation single cropping group, the proportions of Sphingomonas, Gemmatimonas and Pseudomonas increased. Compared the inter cropping group infected with black shank pathogen with the single cropping group infected with black shank pathogen, the proportions of Sphelomonas, Gemmatimonas and Pseudomonas increased, while the proportion of Alcanivorax decreased. The dominant fungi groups were Ascomycota, Basidiomycota and Zygomycota. In dominant fungi groups, compared single cropping group infected with root knot 
nematodes with single cropping groups, the proportions of Colletotrichum, Myrothecium, Humicola and Staphylotrichum increased; the proportion of Aspergillus decreased. Compared inter cropping group infected with root knot nematodes with single cropping group infected with root knot nematodes, the proportions of Colletotrichum, Myrothecium, Humicola and Staphylotrichum decreased; the proportion of Aspergillus increased. Auxarthron was discovered for the first time and might become a new biocontrol bacterium. In the experiments of black shank pathogen inoculating, the proportions of Aspergillus from high to low were AB3.2 > AH3.2 > BH3.2. The proportion of Mortierella in the soil sample of AH3.2 was up to 9.95\%, far greater than the proportions of other soil samples. The differences in the proportions of other dominant fungi were not significant. Among all the dominant groups, Proteobacteria (58.82\%) was the most abundant bacterial group. This result is consistent with studies on bacterial diversity of farmland soil at home and abroad. [6] Among them, Sphingomonas is one of the most effective microorganisms which can degrade soil toxic substances. It can promote the absorption of nutrition, and resist infection by various pathogenic bacteria. Some studies suggest that some strains of Sphingomonas play an important role in nitrogen fixation, dehydrogenation and maintaining the nitrogen balance of soil. [7] Pseudomonas is a dominant genus in the nicotine metabolism of environmental microorganisms. It can produce a variety of plant hormones through nitrogen fixation, so as to promote the absorption of water and mineral elements of tobacco plants; it is also an important phosphorite-dissolving bacterium in the rhizosphere soil of tobacco.[8] Chaetomium can promote the normal growth of root system.[9] Aspergillus is a kind of internal parasitic fungus which can parasite on nematodes through spores; it can produce toxin and promote the formation of nematode killing materials, thus improving the effect of nematode control or even killing nematodes. [10] Meanwhile, in the six soil samples, other fungi occupy high proportions, indicating that there are a large number of rare fungi in the rhizosphere soil of tobacco plants.

\section{Acknowledgements}

This paper is supported by National Natural Science Foundation of China (41361056); Science Research Project of Kunming University (XJL15020); The Scientific Research Project of Yunnan Provincial Education Department (2016ZDX096); Joint Special Fundamental Research of Local Universities in Yunnan Province (2017FH001-042, 2017FH001-041); Talent Introduction Program of Kunming University (YJL14005); Open Fund Project of Key Laboratory of Characteristic Biological Resources Development and Utilization in Yunnan Province (GXKZ201712, GXKZ201716).

\section{References}

[1] S.J. Li, marigold and onion intercropping cultivation technology, J. Bulletin of Agricultural Science and Technology. 5 (2009).

[2] L.H. Jia, T. Wang, X. Huo, et al., Study on the ecological coordination and high yield cultivation techniques of marigold onion, J. Heilongjiang Agricultural Sciences. 4 (2006).

[3] L.H. Jia, Z.D. Liu, Y.H. Yang, et al., Ecological cultivation and disease control techniques in marigold planting of Yanshou County, J. Heilongjiang Agricultural Sciences. 2 (2005).

[4] Q.J. Huang, Study on high yield cultivation techniques of pigment marigold and wheat intercropping, J. Inner Mongolia Agricultural Science and Technology. 5 (2010).

[5] Q.J. Huang, F. Wen, C.H. Zhang, Effects of different density and fertilization on the yield of pigment marigold and wheat intercropping system, J. Agricultural Science-Technology and Information. 26 (2016).

[6] Z.Q. Zhao, Y.J. Feng, H.J. Feng, et al., Anaerobic biotransformation of fluoronitrobenzenes and microbial communities in methanogenic systems, J. Journal of Environmental Science and Health, Part A. 49(2014). 
[7] P.N. Chiang, M.K. Wang, C. Y. Chiu, et al., Effects of cadmium amendments on low-molecular-weight organic acid exudates in rhizosphere soils of tobacco and sunflower, J. Environmental Toxicology. 21(2006).

[8] X.Y. Liang, X.M. Wang, S.X. Wu, et al., Analysis of Saccharina japonica transcriptome using the high-throughput DNA sequencing technique and its vanadium-dependent haloperoxidase gene, J. Acta Oceanologica Sinica. 33(2014).

[9] Z.B. Chen, T.Y. Xia, D.K. Wang, et al., Diversity analysis of endophytic fungi in mentha using illumina miseq high-throughput sequencing technology, J. Medicinal Plant. 7(2016).

[10] A.Y. Huang, G.C. Wang, L.W. He, et al., Characterization of small RNAs from Ulva prolifera by high-throughput sequencing and bioinformatics analysis, J. Chinese Science Bulletin. 27(2011). 